\title{
PERMEATION OF HYDROCORTISONE AND HYDROCORTISONE 21-ALKYL ESTERS THROUGH SILICONE RUBBER MEMBRANES - RELATIONSHIP TO REGULAR SOLUTION SOLUBILITY BEHAVIOR
}

\author{
TIMOTHY A. HAGEN* and GORDON L. FLYNN** \\ College of Pharmacy, The University of Michigan, Ann Arbor, MI 48109-1065 (U.S.A.)
}

(Received March 24, 1986; accepted in revised form June 25, 1986)

\section{Summary}

Rates of permeation of $75 \mu \mathrm{m}$ thick silicone rubber membranes by hydrocortisone and six homologous hydrocortisone 21 -alkyl esters, all applied to the membranes in aqueous media, were assessed in small glass diffusion cells. Solubilities of these agents in water and hexane were also determined. The permeability coefficient of hydrocortisone was $7.4 \times 10^{-5} \mathrm{~cm} / \mathrm{hr}$. Permeability coefficients for the esters ranged from $2.3 \times 10^{-3} \mathrm{~cm} / \mathrm{hr}$ (acetate) to $6.4 \times 10^{-1} \mathrm{~cm} / \mathrm{hr}$ (heptanoate). A direct correlation was found between permeability coefficients and lipophilicity but it appears that the heptanoate ester's mass transfer coefficient is substantially boundary layer controlled. Assessment of fluxes of the steroid solutes from their respective saturated aqueous solutions was made. The flux for hydrocortisone through silicone rubber was greater than the flux for the acetate ester. Thereafter, fluxes of the esters from saturated solutions systematically increased to the point where fluxes for the 21-hexanoate and 21-heptanoate esters were 20 times greater than found for hydrocortisone. This suggests that far more steroid can be delivered through a lipid membrane when the steroid is appropriately derivatized. The overall permeability pattern fits behavior expected of a barrier having a lipid membrane bounded by hydrodynamic layers. The pattern of permeability from saturated solutions was predictable using regular solution theory.

\section{Introduction}

Hydrocortisone is the protype member of a large family of anti-inflammatory steroids used in human and animal medicine. These drugs exhibit a wide range of topical potencies. This variability in potency is partly due to actual differences in intrinsic activities, but it also represents differences in the abilities of the steroids to permeate skin, and therefore it is of considerable theoretical and practical interest to know the degree to which relative corticosteroid activities are determined by relative permeabilities. The present study is a first step in addressing this question and involves the permeation of hydrocortisone

\footnotetext{
*Current address: Pfizer Research, Easton Point Road, Groton, CT 06340, U.S.A.

**To whom all correspondence should be addressed.
} 


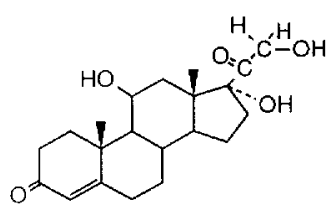

HYDROCORTISONE

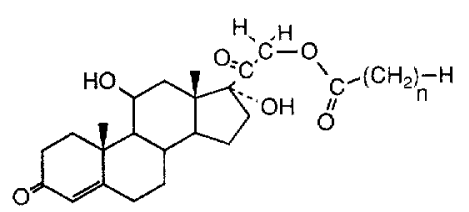

HYDROCORTISONE-21-ESTER

Fig. 1. Structures of hydrocortisone and its 21-esters. The 21-esters are, respectively: $n=1$, acetate; $n=2$, propionate; $n=3$, butyrate; $n=4$, valerate (pentanoate) $n=5$, hexanoate; and $n=6$, heptanoate.

and ester derivatives through a model hydrophobic membrane; it continues our explorations of the influences of chemical structure on the mass transport of drugs across synthetic and biological membranes [1-5]. Concepts here are applicable to the design of drugs, to the design of systems used to administer drugs, to the choice of best drug for a particular route of administration and even to the safety evaluation of drugs and chemicals. Such information is also useful in judging the separation abilities of membranes. The present study with silicone rubber membranes and corticosteroid permeants demonstrates insightfully how the physicochemical properties of a permeant, membrane and medium of application come together to determine the mass transfer profile for structurally rigid nonelectrolytes.

\section{Experimental}

\section{Materials}

Hydrocortisone was obtained as a gift from the Upjohn Company (Kalamazoo, MI) and used as received. All the esters were synthesized from the appropriate anhydride and the provided hydrocortisone. Reagent grade hexane was used in the solubility determinations. Double distilled water was used in all studies.

\section{Synthesis of esters}

Esterification of hydrocortisone at the 21-position proved to be a straightforward, one-step reaction (see Fig. 1 for the structures of hydrocortisone and the esters ). Even in the presence of excess anhydride, the 21-hydroxyl position is preferentially esterified because the $11-\beta$ and 17 -hydroxyl groups are unreactive as the result of internal steric hindrance of the steroid nucleus. The hydrocortisone n-alkyl-21-esters (acetate through heptanoate) were synthesized at room temperature by reacting an excess of each anhydride with $5 \mathrm{~g}$ of hydrocortisone dissolved in $100 \mathrm{ml}$ of neat pyridine. The mole ratio of anhydride to hydrocortisone was set at $5: 1$. Each mixture was vigorously stirred by a tefloncoated magnetic stirring bar as a reaction proceeded. Completion of each reac- 
TABLE 1

Solubilities of hydrocortisone and its 21-esters in water and n-hexane

\begin{tabular}{|c|c|c|c|c|c|c|}
\hline \multirow[t]{2}{*}{ Compound } & \multirow{2}{*}{$\begin{array}{l}\text { Molec- } \\
\text { ular } \\
\text { weight }\end{array}$} & \multirow{2}{*}{$\begin{array}{l}\text { Melting } \\
\text { temper- } \\
\text { ature } \\
\left({ }^{\circ} \mathrm{C}\right)\end{array}$} & \multicolumn{2}{|c|}{ Solubility in water } & \multicolumn{2}{|c|}{ Solubility in hexane } \\
\hline & & & $(\mathrm{mg} / \mathrm{ml})$ & $(\mathrm{mol} / \mathrm{l})$ & $(\mathrm{mg} / \mathrm{ml})$ & $(\mathrm{mol} / \mathrm{l})$ \\
\hline hydrocortisone & 362.5 & 212 & $2.97 \times 10^{-1}$ & $8.19 \times 10^{-4}$ & $3.45 \times 10^{-5}$ & $9.53 \times 10^{-8}$ \\
\hline hydrocortisone 21 -acetate & 404.5 & 224 & $6.29 \times 10^{-3}$ & $1.55 \times 10^{-5}$ & $2.85 \times 10^{-5}$ & $7.03 \times 10^{-8}$ \\
\hline hydrocortisone 21 -propionate & 418.5 & 211 & $1.16 \times 10^{-2}$ & $2.77 \times 10^{-5}$ & $2.11 \times 10^{-4}$ & $5.03 \times 10^{-7}$ \\
\hline hydrocortisone 21 -butyrate & 432.5 & 190 & $7.73 \times 10^{-3}$ & $1.78 \times 10^{-5}$ & $4.19 \times 10^{-4}$ & $9.64 \times 10^{-7}$ \\
\hline hydrocortisone 21 -valerate & 446.5 & 184 & $3.05 \times 10^{-3}$ & $6.78 \times 10^{-6}$ & $1.02 \times 10^{-3}$ & $2.22 \times 10^{-6}$ \\
\hline hydrocortisone 21-hexanoate & 460.5 & 115 & $1.42 \times 10^{-3}$ & $3.06 \times 10^{-8}$ & $3.46 \times 10^{-3}$ & $7.45 \times 10^{-6}$ \\
\hline hydrocortisone 21 -heptanoate & 474.5 & 111 & $9.88 \times 10^{-4}$ & $2.06 \times 10^{-6}$ & $1.24 \times 10^{-2}$ & $2.59 \times 10^{-5}$ \\
\hline
\end{tabular}

"These melting points are in close agreement with literature values [21].

tion was followed by thin-layer chromatography (chloroform: methanol, 9:1 and silica gel TLC plates with fluorescent indicator; Eastman Kodak Company, Rochester, NY).

Approximately one hour after hydrocortisone could no longer be detected by TLC, liberal amounts of cold $0.1 \mathrm{~N}$ sulfuric acid were added to a reaction mixture to solubilize the pyridine but precipitate the ester, and crude crystals of the product were collected on a buchner funnel. In the synthesis of the longer chain length hexanoate and heptanoate esters it was also necessary to wash the crystals with $0.1 \mathrm{~N} \mathrm{NaOH}$ in order to remove the long chain carboxylic acid formed in 1:1 ratio as a by-product of the reaction. The hydrocortisone 21esters were recrystallized at least two times from either methanol/water (acetate through valerate) or hexane/methylene chloride (hexanoate and heptanoate) solution. Large crops of white crystalline material were obtained in all instances. Crystals were dried under vacuum for $24 \mathrm{hr}$ to remove residual solvent. The overall yields were approximately $80 \%$.

Infrared spectra of the esters clearly showed the added ester linkage. Ester melting points were determined on a conventional melting point apparatus and were in close agreement with literature values. However, decomposition was observed to occur upon melting under ambient atmospheric conditions. The melting temperatures and molecular weights for hydrocortisone and the 21esters are listed in Table 1.

\section{Membranes}

Fillerless silicone polymer membranes were prepared for the studies. Polydimethylsiloxane polymer base (gift from Dow Corning, Midland, MI) without silica filler was mixed with stannous octanoate (Catalyst M, Dow Corning, Midland, MI), approximately $10 \mathrm{~g}$ of base to $0.1 \mathrm{~g}$ of catalyst. This proportion allows 10 min working time with the soft mixture. The mixture was degassed for $3 \mathrm{~min}$, after which an excess was spread about a $50 \mu \mathrm{m}$ metal spacer. The mass was pressed flat with a hydraulic press, uniformly spreading the mixture 
between the spacers. The membranes were allowed to polymerize for several hours (vulcanization took $\sim 30 \mathrm{~min}$ ). Sheets of cellophane were placed beneath and above the polymer mass before it was pressed flat to facilitate separation from the press's surfaces. The cellophane was easily removed after vulcanization.

Transparent, homogeneous silicone membranes were obtained and carefully cut from the sheet. The fillerless membranes proved to be fragile but were workable. The membranes were uniformly $0.0075 \mathrm{~cm}$ thick as determined with a micrometer. The membranes were soaked and rinsed in distilled water for several hours before use. In all studies, the only solvent in contact with the membranes was double distilled water.

\section{Assay method}

Reverse phase HPLC was chosen as the analytical procedure because it provided a means of simultaneously separating and quantifying the steroids. A liquid chromatographic system (Waters Associates Inc, Milford, MA) was employed with a UV/VIS absorbance detector. Suitable chromatographic systems were developed in which a mixed methanol: water solvent ( $80: 20$ to $60: 40$ depending on the steroid) and a $\mu$ Bondapak $\mathrm{C}-18$ column served as the mobile and stationary phases, respectively. The absorbance of steroids was measured at $254 \mathrm{~nm}$.

Standard solutions of hydrocortisone and the esters were prepared in methanol for calibration of chromatographic peak heights. Injection volumes for the standard and unknown solutions were equivalent. As internal standards, appropriate hydrocortisone $\mathrm{n}$-alkyl-21-esters were used. The sensitivity range for the chromatographic system was determined to be about $0.01 \mu \mathrm{g} /$ sample to $10 \mu \mathrm{g} / \mathrm{sample}$ for all the analogs. Standard curves demonstrated excellent linearity over the entire sensitivity range. Standard peak heights were determined daily for quantification of unknown solutions and the experimental error for the standard solutions was consistently less than $2 \%$. The reproducibility of the chromatographic system was excellent over long periods of time.

A chromatogram of hydrocortisone and the six esters is shown in Fig. 2, illustrating the chromatographic identification of each homolog. An interesting point is the relative distances between the chromatographic peaks. A nearly linear relationship is found when log (normalized elution volume) is plotted against chain length. Since reverse-phase chromatography involves partitioning of the solute between the C-18 column and the methanol: water solvent system, such partitioning-based chain length dependencies are to be expected. Despite the small quantities of steroids involved, the HPLC method of analysis proved to be reproducible and suitable for the mass transfer studies.

\section{Solubility determinations}

The solubilities of hydrocortisone and the esters in water and hexane were obtained by equilibrating large excesses of solute (greater than $0.5 \mathrm{~g}$ ) with the 


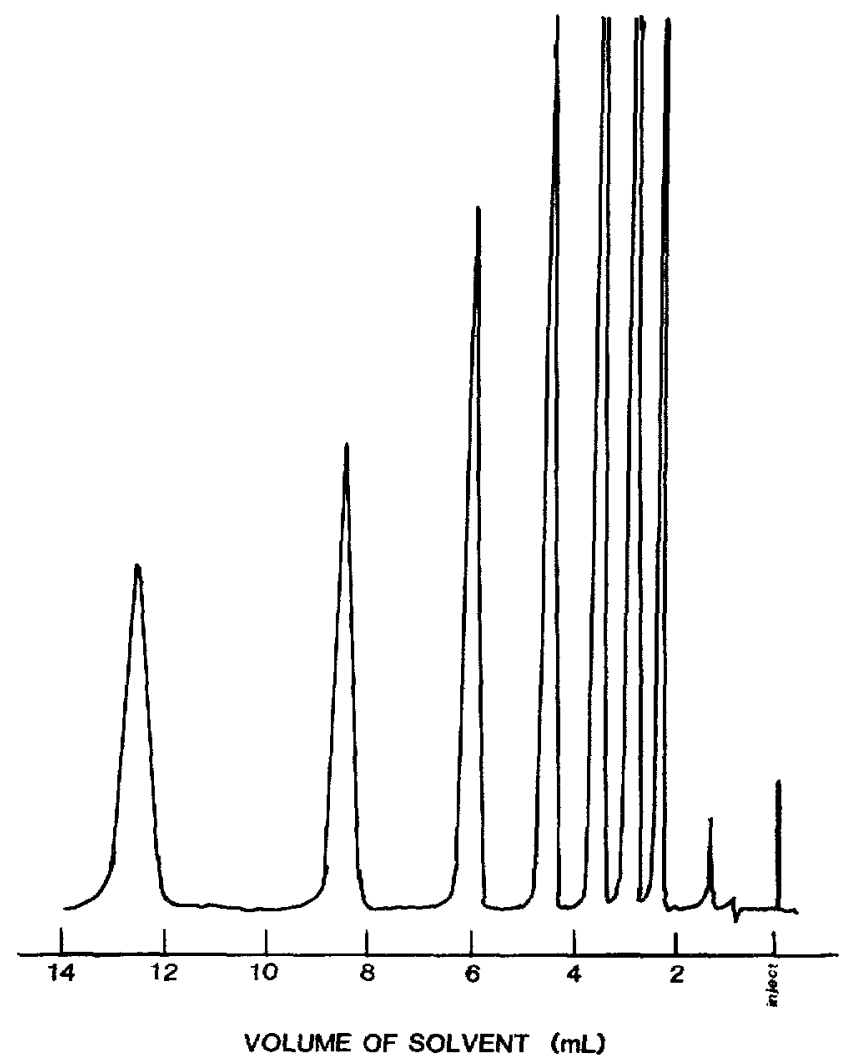

Fig. 2. Tracing of HPLC chromatogram for a sample containing arbitrary amounts of hydrocortisone and its acetate through heptanoate esters. The major peaks are, from right to left, hydrocortisone (retention time $\sim 2.5 \mathrm{~min}$ ), hydrocortisone 21 -acetate $(\sim 3 \mathrm{~min}$ ), hydrocortisone 21 propionate ( $\sim 3.7 \mathrm{~min}$ ), hydrocortisone 21 -butyrate $(\sim 4.6 \mathrm{~min})$, hydrocortisone 21 -valerate $(\sim 6.2$ $\mathrm{min}$ ), hydrocortisone 21-hexanoate $(\sim 8.6 \mathrm{~min})$ and hydrocortisone 21-heptanoate $(\sim 13 \mathrm{~min})$. The $70: 30$ methanol : water solvent flow rate was $1 \mathrm{ml} / \mathrm{min}$ and the separation was on a $\mu$ Bondapak C-18 column.

neat solvents in covered, jacketed glass containers [6]. The temperature was maintained at $25^{\circ} \mathrm{C}$ by a constant temperature water bath. Vigorous stirring was supplied by teflon-coated magnetic stirring bars. An excess of solute was present in all the slurries.

The solubilities of hydrocortisone and the short chain esters in hexane were so low that large volumes of hexane $(300-600 \mathrm{ml})$ had to be equilibrated with excess solute for periods extending beyond 10 days. Large aliquots of these slurries were passed through $0.22 \mu \mathrm{m}$ pore size filters (Fluoropore filters, Millipore Corp., Bedford, MA). The volume of the collected supernatant was measured and then brought to dryness with the aid of an evaporator. The procedure for the more soluble higher esters was the same except smaller sample volumes 
were used. Collected steroid was reconstituted in $3 \mathrm{ml}$ of methanol already containing the internal standard, which was hydrocortisone 21-butyrate in most instances. As a general procedure, concentrations were determined three times on each sample and averages were taken. The solubility in hexane for each homolog represents the average of three independent solubility determinations.

To determine the solubilities in water, concentrations in the supernatant were followed as a function of time until concentration plateaus were established. Several samples were taken thereafter and averages were calculated from the plateau values. The entire procedure was repeated for each compound and thus each recorded solubility value represents the average of two equilibrium averages from independent determinations.

\section{Diffusion cell}

Small diffusion cells were prepared from tempered glass in local shops. These were in the form of two cylindrical half-cells each having an experimental halfcell volume of $1.6 \mathrm{ml}$. Each half-cell had two ports, one for sampling and one to accommodate the shaft of the stirring device. The two half-cells with membrane between were rigidly clamped together with a spring clamp. The essential physical characteristics of the diffusion cell are described in earlier publications from these labs [5,7]. Similar diffusion cells have been successfully used in previous studies involving silicone rubber membranes $[1,8]$.

The cell body was completely immersed in a constant temperature bath. The seal between membrane and glass was secure and there was no leakage. The cell ports protruded above the surface of the water bath, allowing for sampling and stirring. The contents of each half-cell were stirred at $150 \mathrm{rpm}$ by constant speed motor (Model CA, 115 V, Hurst Inc., Princeton, IN). Thin glass rods were attached to the motors by bits of tightly fitting tubing and these drove the teflon stirring propellers inside the cell. The stirring adequately mixed the donor solutions and suspensions without causing visible disturbance of the membrane. The area available for diffusion between compartments was 0.636 $\mathrm{cm}^{2}$.

\section{Experimental determination of permeability coefficients}

The experimental determinations of the steady-state fluxes of the steroids through the silicone rubber membranes proved to be a challenging problem for the hydrocortisone series. Due to large differences in physicochemical properties within the series and given the limits of sensitivity of the HPLC system, it was impossible to determine steady-state fluxes for the entire series using a single experimental procedure. In all studies, however, the stirring rate was kept at $150 \mathrm{rpm}$, the temperature was maintained at $25^{\circ} \mathrm{C}$, the donor and receiver solvents were double distilled water, the area of the membrane was $0.636 \mathrm{~cm}^{2}$ and the thickness of the membrane was $0.0075 \mathrm{~cm}$.

Hydrocortisone and esters from acetate through butyrate permeate silicone 
rubber at slow rates. The experimental procedure for these compounds and the valerate ester was chosen to maximize the permeation rate and the experimental sensitivity. Large excesses of each drug were equilibrated with water at $25^{\circ} \mathrm{C}$ and the saturation concentration of the slurry was confirmed by previous methods. The saturated and concentrated suspensions were transferred to the donor compartment. During a diffusion run, the donor contents were exchanged daily with fresh suspension to obviate problems associated with bacterial growth within the media. The donor compartment's contents were frequently sampled and there was no occasion where there was a detectable departure from saturation during the course of an experiment.

The entire receiver contents were withdrawn at set time intervals selected to insure that sink condition existed throughout each experiment. Each sampling was followed by two total rinses of the receiver. The three aliquots were pooled in a single tapered $15 \mathrm{ml}$ test tube and brought to dryness using a microflash evaporator. Additional samples and their rinses were added to the same test tube until a sufficient amount of drug was present in the test tube for reliable assay. The drug was then reconstituted in $200 \mu \mathrm{l}$ of methanol containing, as an internal standard, a non-interfering hydrocortisone 21 -ester, and the contents were vortexed for at least $30 \mathrm{~min}$. Samples $(10-100 \mu \mathrm{l})$ were drawn for analysis by HPLC, the sample volume depending on the compound.

Possible decomposition into hydrocortisone was monitored in the receiver compartment throughout the course of each experiment. Irrespective of the ester, the quantities of free hydrocortisone were analytically insignificant. Trial runs of the entire assay procedure were performed beginning with known, trace amounts of hydrocortisone mixed with its esters. Recovery of the intact esters and hydrocortisone was reproducible and essentially complete. It was never less than $90 \%$ of known amounts.

The solubilities of the hexanoate and heptanoate esters were too low to successfully apply the receiver accumulation method. However, the permeability rates of hydrocortisone 21-valerate, 21-hexanoate and 21-heptanoate were sufficiently fast that first-order rates of depletion from the donor department to a sink could be measured in a reasonable period of time. Therefore, stock solutions of drug were freshly made for each experiment and placed in the donor compartment of the cell. This compartment was rinsed twice with stock solution, then the initial concentration was determined. Appropriate sampling intervals were selected for each ester such that a $10 \%$ or greater depletion of the donor concentration was measured at the end of each sampling interval. After each sample was taken from the diffusion cell, the remaining donor compartment solution was removed, the compartment was rinsed and then filled with fresh stock solution at the original concentration. The assay method, which evidences free hydrocortisone when it is present, indicated that hydrolysis of the ester to hydrocortisone did not occur to a significant extent within the time 
course of the runs. The receiver compartment was continuously flushed with water over the course of the experiments to maintain the sink condition.

\section{Data reduction}

Experimental data is presented in Fig. 3 illustrating the method of determination of the permeability coefficients of hydrocortisone and esters from acetate through valerate. In order to obtain sufficient hydrocortisone 21 -acetate for a single assay, a time interval of $48 \mathrm{hr}$ was required. The total amount of drug collected every $48 \mathrm{hr}$ (two $24 \mathrm{hr}$ samples) was approximately $0.5 \mu \mathrm{g}$. Roughly $1.0 \times 10^{-2} \mu \mathrm{g}$ entered the receiver compartment every hour.

Permeability coefficients for the receiver accumulation procedure were calculated for each sample from the following expression:

$$
P=\frac{V_{\mathrm{R}}(\mathrm{d} C / \mathrm{d} t)}{A(\Delta C)}
$$

where $V_{\mathrm{R}}$ is the volume of the receiver compartment, $1.6 \mathrm{ml} ; A$ is the area of the exposed membrane, $0.636 \mathrm{~cm}^{2}$; and $V_{\mathrm{R}}(\mathrm{d} C / \mathrm{d} t)$ is the steady-state flux through the total barrier. The value of $\mathrm{d} C / \mathrm{d} t$ was determined by taking the ratio of the tatal amount permeated in an interval of time to the length of the time interval. The term $\Delta C$ is the concentration differential across the membrane. This was effectively equal to the saturation concentration in the donor as, through total exchange sampling, a near zero receiver concentration (sink condition) was closely approximated. The number of samples correspond to the number of peaks shown in Fig. 3. None of the compounds evidenced detectable lag times within the extended time frames of the experiments.

The experimental method for the permeability coefficient determinations of the valerate, hexanoate, and heptanoate esters of hydrocortisone is illustrated in Fig. 4 with experimental data for hydrocortisone 21-heptanoate. The change in the donor concentration with respect to time is given by the following equation:

$\ln \frac{C_{\mathrm{t}}}{C_{0}}=-k t$

where $k$ is the rate constant for the first order disappearance, $C_{0}$ is the donor concentration at time zero, and $C_{t}$ is donor concentration at time $t$. The permeability coefficient is obtained from:

$$
P=k \frac{V_{\mathrm{D}}}{A}
$$

where $V_{\mathrm{D}}$ is the donor compartment volume $(1.4 \mathrm{ml})$. Results for sequential intervals were averaged to get the final permeability coefficient values. 


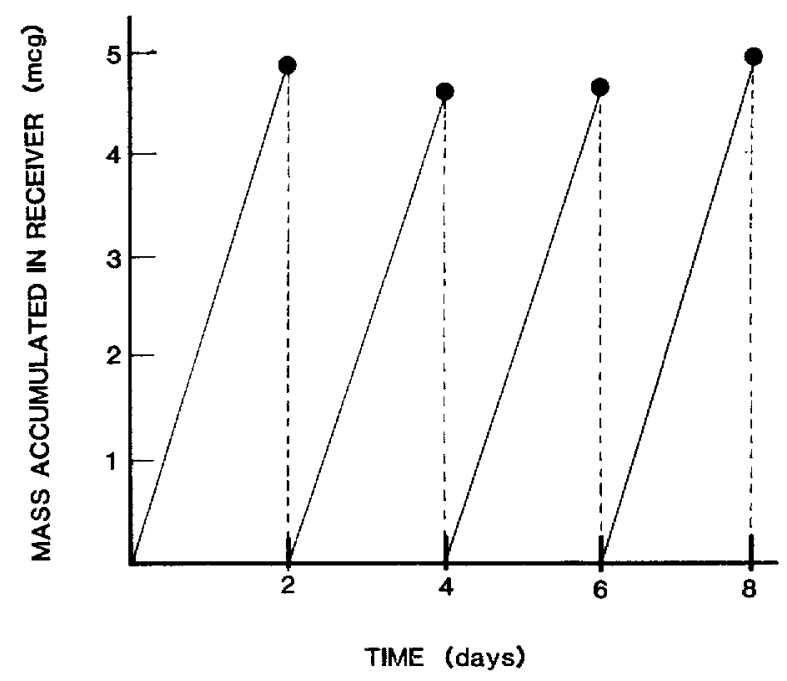

Fig. 3. Plot showing the cumulative amounts of hydrocortisone 21-acetate which penetrated a silicone rubber membrane over two-day periods in a continuous experiment running eight days. The permeability coefficient for a run was the average of the permeability coefficients calculated for all sub-intervals in the run.

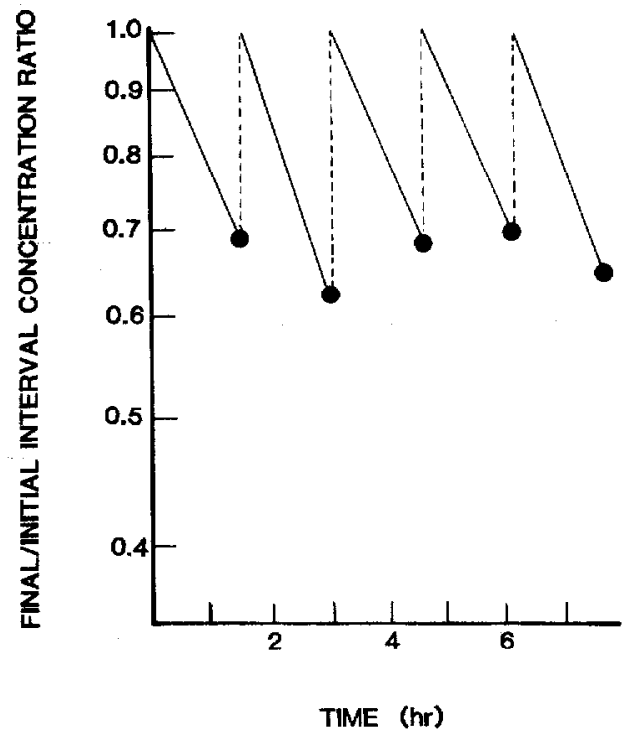

Fig. 4. Semilogarithmic plot illustrating the donor depletion method used to obtain permeability coefficients for the higher esters. The particular data are for a run with hydrocortisone 21-heptanoate. In this instance sampling intervals were 1.5 hours in duration, at which point the ester concentration drops $33-38 \%$ of its initial value. The permeability coefficient assigned to a run was the average of the individual permeability coefficients for the $1.5 \mathrm{hr}$ sub-intervals. 


\section{TABLE 2}

Permeability coefficients for hydrocortisone and hydrocortisone n-alkyl 21 -esters at $25^{\circ} \mathrm{C}$ through fillerless silicone membrane with thickness of $0.0075 \mathrm{~cm}$

\begin{tabular}{llll}
\hline Compound & $\begin{array}{l}\text { Number } \\
\text { of runs }\end{array}$ & $\begin{array}{l}\text { Number of } \\
\text { experiments }\end{array}$ & $\begin{array}{l}\text { Permeability } \\
\text { coefficient and } \\
\text { std dev. (cm/hr) }\end{array}$ \\
\hline $\begin{array}{l}\text { Results from receiver assay: } \\
\quad \text { Hydrocortisone }\end{array}$ & 1 & 4 & $7.4( \pm 0.8) \times 10^{-5}$ \\
Hydrocortisone 21-acetate & 1 & 4 & $2.3( \pm 0.08) \times 10^{-3}$ \\
$\quad$ Hydrocortisone 21-propionate & 1 & 5 & $5.8( \pm 0.5) \times 10^{-3}$ \\
Hydrocortisone 21-butyrate & 1 & 5 & $2.2( \pm 0.4) \times 10^{-2}$ \\
$\quad$ Hydrocortisone 21-valerate & 1 & 4 & $8.5( \pm 1.0) \times 10^{-2}$ \\
Results from donor assay: & 2 & 6 & $9.7( \pm 1.0) \times 10^{-2}$ \\
$\quad$ Hydrocortisone 21-valerate & 2 & 5 & $4.5( \pm 0.2) \times 10^{-1}$ \\
Hydrocortisone 21-hexanoate & 2 & 5 & $6.4( \pm 0.2) \times 10^{-1}$ \\
Hydrocortisone 21-heptanoate & 2 & &
\end{tabular}

\section{Results}

Solubility data and appropriate supportive data for hydrocortisone and its homologous 21-esters are given in Table 1 . Solubility is expressed both in $\mathrm{mg} / \mathrm{ml}$ and $\mathrm{mol} / \mathrm{l}$ units.

Permeability coefficients are summarized in Table 2 . It should be noted that the permeability coefficient for the valerate was determined by each of the methods. The values are in good agreement and an average value of $9.1 \times 10^{-2}$ $\mathrm{cm} / \mathrm{hr}$ was used in the following data analysis.

\section{Discussion}

In designing drug delivery systems one is generally faced with the problem of choosing a drug from a group of pharmacologically comparable compounds which best meets route dependent activity and drug delivery requirements. Membrane permeation factors can be deciding factors. In the absence of experimental data on permeability, the choice of the drug might be based on chemical structure but to do so one has to have a clear idea of the absorption determining structural features. T. Higuchi [9] discussed the issues here with regard to drug delivery through the skin and pointed out that thermodynamic concepts can be applied to compound selection because a drug's thermodynamic activity within its formulation determines its rate of absorption. The maximum attainable activity and thus the maximum attainable flux are achieved when the drug is saturated in its vehicle. The drug which has the highest relative activity in its saturated state may be the best drug from the drug delivery standpoint. The activity of a saturated crystalline organic solid 
is determined by intracrystalline association, which is dependent on bonding groups and also on the spacial arrangement of the molecules. Molecular packing often changes dramatically and in unpredictable ways with molecular modification and thus relative thermodynamic potentials of saturated analogs and derivatives can and do vary appreciably. Limiting mass transfer rates generally directly parallel relative activities. Since classical thermodynamics does not provide a means of ordering thermodynamic activities of different substances on a common scale, Higuchi [9] suggests that we use the solubilities of analogs in apolar hydrocarbon solvents as the measure of relative activity. This has been done in the present work. While activity coefficients are not set forth explicitly, they have been effectively incorporated using regular solution theory. The theoretical generalizations of Higuchi [9] are borne out.

Before analyzing the data in the above regards, an early and unusual observation in the research is worthy of note. The present studies were begun with commercial $0.0127 \mathrm{~cm}$ thick silicone rubber sheeting (Silastic 372 Medical Grade Sheeting, Dow Corning, Midland, MI) . A $25^{\circ} \mathrm{C}$ permeability coefficient for hydrocortisone of $2.0 \times 10^{-5} \mathrm{~cm} / \mathrm{hr}$ was determined. However, exceptionally long lag times, in excess of $70 \mathrm{hr}$, were also observed. Exhaustive extraction of the membranes with methylene chloride immediately after the diffusion experiments drew out large amounts of hydrocortisone. The membrane/water partition coefficient calculated from the extracted material was on the order of unity. Using a previously determined value for the diffusivity of hydrocortisone in polydimethylsiloxane, $1.62 \times 10^{-3} \mathrm{~cm}^{2} / \mathrm{hr}[6,10]$ and the observed partition coefficient (1.0) yielded a permeability coefficient, $K D / h$, greater than $1 \times 10^{-1} \mathrm{~cm} / \mathrm{hr}$, a value which is orders of magnitude larger than experimentally determined.

Commercial silicone sheeting contains a collodial silica filler and the adsorption of permeants to the surface of this filler during their diffusive transit has been reported [11-13]. Roseman [11] indicated that apparent membrane/ water partition coefficients for selected steroids were increased by the presence of the relatively coarse filler material found in Silastic ${ }^{\mathrm{TM}} 382$ [7]. The partition coefficient of 17-hydroxyprogesterone was increased 2.5-fold over that found for fillerless silicone rubber. Flynn and Roseman [12] reported that the extent of filler adsorption increased with increasing polarity of alkyl p-aminobenzoates test permeants, a trend also followed by the steroids studied by Roseman.

Higuchi and Higuchi [14], Paul and Kemp [13], Most [15] and Flynn and Roseman [12] have demonstrated that adsorption lengthens lag times in proportion to the amount of permeant adsorbed. The exaggeratedly long lag time found when hydrocortisone permeated the Silastic ${ }^{\mathrm{TM}} 382$ [7] membranes was totally inconsistent with the known diffusivity of this compound in polydimethylsiloxane and led to the suspicion that hydrocortisone was extensively bound to the filler. Inconsistencies in membrane/water and hexane/water par- 
titioning also supported this view. Analysis of the permeability data and solubility data together indicated that for every hydrocortisone molecule which was free and mobile in the silicone rubber about 1000 hydrocortisone molecules are immobilized via adsorption. The steady-state permeation rate thus was low not because of low diffusivity but because of a very small true silicone/water partition coefficient (estimated to be about $2.4 \times 10^{-4}$ ). To eliminate fillerrelated complications as these, fillerless silicone rubber membranes were made and used in the remainder of the experiments. Here hydrocortisone's steadystate permeation rate was consistent with that found for the commercial membranes containing filler but, at the same time, lag times were so short that they could not be experimentally distinguished from zero in experiments with $24 \mathrm{hr}$ sampling periods.

Based on previous work involving the permeation of polydimethylsiloxane membranes by alkyl $p$-aminobenzoates [1], it was reasoned that permeability coefficients through a hydrocortisone ester series spanning six methylene units might reflect the influences of both the membrane and hydrodynamic boundary layers. The total diffusional resistance would be the sum of the resistances of the hydrodynamic layers on each side of the membrane and the resistance of the silicone rubber membrane. It has been shown that the steady-state permeability coefficient, $P$, for the membrane-boundary layer series barrier takes the general form [16]:

$P=\frac{D_{\mathrm{m}} D_{\mathrm{aq}} K_{\mathrm{m} / \mathrm{aq}}}{h_{\mathrm{m}} D_{\mathrm{aq}}+\sum h_{\mathrm{aq}} D_{\mathrm{m}} K_{\mathrm{m} / \mathrm{aq}}}$

where $D$ s are diffusivities, $h \mathrm{~s}$ are thicknesses and $K_{\mathrm{m} / \mathrm{aq}}$ is the membrane/water partition coefficient. The subscripts ' $m$ ' and 'aq' refer to the membrane and the aqueous boundary layer. At the same time, partitioning of homologs between aqueous and hydrophobic media follows the relationship [16]:

$\left(K_{\mathrm{m} / \mathrm{aq}}\right)_{n}=\left(K_{\mathrm{m} / \mathrm{aq}}\right)_{0} 10^{\pi n}$

where $n$ is the homolog chain length, $\left(K_{\mathrm{m} / \mathrm{aq}}\right)_{0}$ is the hypothetical partition coefficient at $n=0$ (an intercept value) and $\pi$ is the methylene increment obtained as the slope of a plot of $\log \left(K_{\mathrm{m} / \mathrm{aq}}\right)_{n}$ versus $n$. Figure 5 shows that partitioning of the hydrocortisone homologs between hexane and water follows the expected exponential pattern. Past work with alkyl $p$-aminobenzoates established parallels in hexane/water and silicone oil/water partitioning behavior [17]. It follows that:

$P=\frac{D_{\mathrm{m}} D_{\mathrm{qa}}\left(K_{\mathrm{m} / \mathrm{aq}}\right)_{0} 10^{\pi n}}{h_{\mathrm{m}} D_{\mathrm{aq}}+\sum h_{\mathrm{aq}} d_{\mathrm{m}}\left(K_{\mathrm{m} / \mathrm{aq}}\right)_{0} 10^{\pi n}}$

This suggests that permeability coefficients should be directly dependent on the partition coefficient when $n$ is small and should be partitioning independent when $n$ is large. 


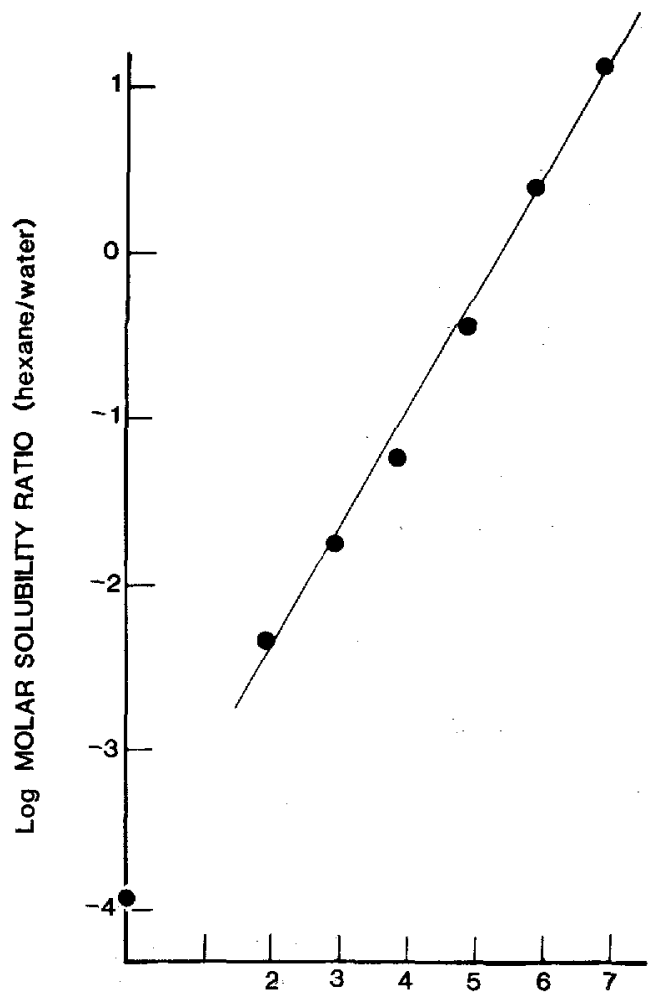

ALKYL CHAIN LENGTH

Fig. 5. Semilogarithmic plot showing the hexane/water partition coefficients of hydrocortisone (arbitrarily plotted on $y$-axis at $n=0$ ) and its 21-alkyl esters. It can be seen that the partition coefficients of the esters increase exponentially with increasing alkyl chain length. The ratios of experimentally determined molar solubilities in hexane and water are taken as the partition coeflicients.

An estimate of the total effective thickness of the boundary layers, $\sum h_{\text {aq }}$, can be made in order to establish an upper limit for the attainable permeability coefficient in this system. The aqueous diffusion coefficient for hydrocortisone should be near $2.5 \times 10^{-7} \mathrm{~cm}^{2} / \mathrm{sec}$, a value reported by Flynn et al. [18] for progesterone, since progesterone and hydrocortisone are roughly the same molecular size. Given the relative lack of diffusivity sensitivity to molecular volume in liquid media, even the addition of the ester linkages should not profoundly lower $D_{\mathrm{aq}}$ and the value of $2.5 \times 10^{-7} \mathrm{~cm}^{2} / \mathrm{sec}$ can be considered representative for the entire hydrocortisone series. The effective aqueous boundary thickness for this diffusion cell assembly at a stirring rate of $150 \mathrm{rpm}$ has been determined from the limiting permeability coefficient of homologous alkanols by Behl and Flynn (unpublished results) to be $0.025 \mathrm{~cm}$. Substitution of these estimates for $D_{\mathrm{aq}}$ and $h_{\mathrm{aq}}$ into eqn. (6) under the circumstances that 


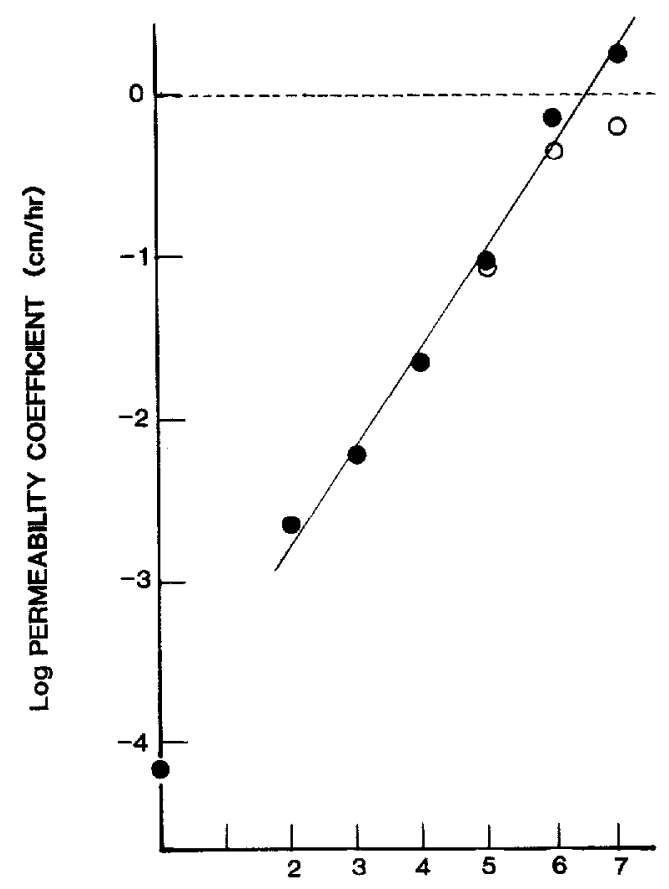

ALKYL CHAIN LENGTH

Fig. 6. Semilogarithmic plot of experimentally derived permeability coefficients versus alkyl chain length of the esters. Hydrocortisone's value is plotted on the $y$-axis at $n=0$ though it is not a homolog. The open circles evident for the 21-valerate, 21-hexanoate and 21-heptanoate are the actual data. The filled-in circles for all compounds are permeability coefficients corrected to remove the influence of the hydrodynamic boundary layers. This correction was without measurable effect on the permeability coefficients of hydrocortisone and its 21-esters through the butyrate. The dotted line on the graph indicates the boundary layer control limit determined previously using alkanols. It can be seen that, after the boundary layer correction is made, the permeability coefficients increase systematically and exponentially even as partition coefficients increase.

$\sum h_{\mathrm{aq}} D_{\mathrm{m}}\left(K_{\mathrm{m} / \mathrm{aq}}\right)_{0} 10^{\pi n} \gg h_{\mathrm{m}} D_{\mathrm{aq}}$ gives $1.0 \mathrm{~cm} / \mathrm{hr}$ as the limiting value for the mass transfer coefficient as the membrane/water partition coefficient becomes large (boundary layer control value).

The experimental permeability coefficients (Table 2) can be linearized to partition coefficient dependent, pure membrane values by factoring out this boundary layer resistance. Both experimental and corrected values for the permeability coefficients are plotted in Fig. 6 on a semilogarithmic scale as a function of chain length. From the positions of the boundary layer adjusted points it is clear that the hexanoate and heptanoate esters are under the influence of the boundary layers. When the boundary layer influence is factored out, all ester permeability coefficients fit well to an exponentially increasing pattern. Regression analysis for the line drawn through the boundary layer 
normalized data yields a slope of 0.61 , the methylene increment for permeability, and a $y$-intercept of -4.0 . Providing diffusivities in polydimethylsiloxane are little affected by the chain length increases, the methylene increment of permeability for these steroid esters should be essentially the same as the $\pi$-value for their silicone rubber/water partitioning. The $\pi$-value from hexane/water partitioning determined from Fig. 4 is 0.698 . The difference in these $\pi$-values, 0.09 , is larger than might be anticipated given the similarities of the media and may indicate that diffusivity does decrease some through the series.

In the absence of boundary layer influence, the steady-state flux per unit area, $J_{\mathrm{ss}}$, of an ester diffusing from a saturated aqueous solution, through the membrane and into a receiver sink is simply the product of the true membrane permeability coefficient, $P_{\mathrm{m}}$, and the aqueous solubility. Thus:

$J_{\mathrm{ss}}=P_{\mathrm{m}} S_{2, \mathrm{aq}}$

where $S_{2, a q}$ is the molar solubility of the permeant in water. The true membrane permeability coefficient may also be defined as:

$P_{\mathrm{m}}=\frac{D_{\mathrm{m}} K_{\mathrm{m} / \mathrm{aq}}}{h_{\mathrm{m}}} \cong \frac{D_{\mathrm{m}}}{h_{\mathrm{m}}} \frac{S_{2, \mathrm{~m}}}{S_{2, \mathrm{aq}}}$

where $S_{2, \mathrm{~m}}$ is the molar solubility of the permeant in the membrane, and thus:

$J_{\mathrm{ss}} \cong \frac{D_{\mathrm{m}}}{h_{m}} S_{2, \mathrm{~m}}$

Thus, the boundary layer adjusted flux determined from external saturated solutions of these steroids should increase exactly as their solubilities in the membrane increase, at least to the extent that $D_{m}$ is invariant and the boundary layers are uninvolved.

Polydimethylsiloxane is a low cohesive energy density material. Michaels et al. [19] have estimated its solubility parameter to be $7.6\left(\mathrm{cal} / \mathrm{cm}^{3}\right)^{1 / 2}$ using Small's molar attraction constants [20]. The value for hexane is similar, namely $7.27\left(\mathrm{cal} / \mathrm{cm}^{3}\right)^{1 / 2}$. The likeness in the solvent properties of these two media is seen in the strikingly parallel solubilities of the $\mathrm{n}$-alkyl $p$-aminobenzoates within them [17]. In a previous study we have shown that hydrocortisone's solubility in hexane fits the general expectations of regular solution theory [6]. It seems reasonable that the solubilities of its esters in the silicone rubber matrix should also follow regular solution patterns of behavior. The solubilities for the hydrocortisone series in polydimethylsiloxane can be estimated on this basis and theoretically factored into the permeation results. Taking this approach, the mole fractional solubilities for hydrocortisone and all of its esters were calculated using the previously determined solubility parameter value of 12.4 for hydrocortisone and ester solubility parameters derived therefrom using molar attraction constants [6]. These derived solubility parameters were, in turn, 
used to estimate ideal solubilities with the aid of the hexane solubility data. Mole fraction solubilities in the silicone rubber polymer were then calculated and converted to $\mathrm{mol} / \mathrm{cm}^{3}$. This allowed fluxes in units of $\mathrm{mol} / \mathrm{cm}^{2}-\mathrm{hr}$ to be calculated. Since the silicone rubber used in these studies is almost entirely composed of polymer chains consisting of repeating dimethylsiloxy units with a molecular weight of 74 , a solvent molecular weight of 74 was used for the polymer in the theoretical calculations. The mole fractional solubilities were converted to molar concentrations by the following expression:

$S_{2, \mathrm{~m}}=X_{2, \mathrm{~m}} \frac{1000}{M_{1} d_{1}}$

where $M_{1}$ is the molecular weight of the dimethylsiloxy unit and $d_{1}$ is the density of the polymeric material. The experimentally determined density for fillerless polydimethylsiloxane was 0.84 . All derived data are given in Table 3 .

Steady-state fluxes from saturated aqueous solutions were predicted from eqn. (9) and the theoretically estimated solubilities in silicone rubber (Table $3)$. Since the actual value of the ratio $D_{\mathrm{m}} / h_{\mathrm{m}}$ is unknown, the solubility estimate for hydrocortisone 21-acetate in polydimethylsiloxane was assumed real, which provided an arbitrary reference value for $D_{\mathrm{m}} / h_{\mathrm{m}}$ of $5.9 \times 10^{-2} \mathrm{~cm} / \mathrm{hr}$. The predicted fluxes of the other esters were, in this way, normalized to hydrocortisone 21-acetate's. In Fig. 7 predicted fluxes are compared with the experimental fluxes, which appear both as the experimental values and as experimental values corrected for the boundary layer influence. An increase greater than 100-fold is projected in flux from the acetate to the heptanoate ester but only a little over a 50 -fold increase is actually seen. Nevertheless, regular solution flux predictions roughly agree with the experimental values and, more importantly, the projected shape of the theoretically determined pattern is the same as that of the experimental data. There is a systematic, gradual departure of the regular solution predictions from the actual trend such that a relatively large discrepancy is seen for the heptanoate ester. This, of course, is in part due to the fact that the heptanoate is the most distant from the acetate ester, the reference point to which the curve is normalized. Given the several tiers of assumptions, the overall fit to regular solution theory is satisfying. The failure of the approach to give an exacting fit to the data is in part rooted in the approximate nature of the molar attraction constants and partial molar volumes used in the analysis. Small errors in these magnify upon repetitive additions of the same moiety $\left(\mathrm{CH}_{2}\right)$ to the molecule. It is of particular interest that the saturated state flux of hydrocortisone 21 -acetate is less than hydrocortisone's saturated state flux despite the fact that hydrocortisone is considerably more polar. The regular solution approach predicts this order for the two fluxes, amongst other aspects supporting Higuchi's [9] idea that solubility in the membrane is generally the discriminating factor in rate determination. 
TABLE 3

Estimated solubility parameters of hydrocortisone 21-esters and also estimated solubilities of hydrocortisone and its 21 -esters in polydimethylsiloxane

\begin{tabular}{lllllll}
\hline Compound & $\begin{array}{l}V_{\mathrm{m}}^{\mathrm{a}} \\
\left(\mathrm{cm}^{3}\right)\end{array}$ & $\begin{array}{l}\left(E V_{\mathrm{m}}\right)^{1 / 2^{\mathrm{b}}} \\
\left(\mathrm{cal} \cdot \mathrm{cm}^{3}\right)^{1 / 2}\end{array}$ & $\begin{array}{l}\delta_{2}^{\mathrm{c}} \\
\left(\mathrm{cal} / \mathrm{cm}^{3}\right)^{1 / 2}\end{array}$ & $\begin{array}{l}\Delta H_{\mathrm{f}}^{\mathrm{d}} \\
(\mathrm{kcal})\end{array}$ & $X_{2, \mathrm{~m}}^{\mathrm{e}}$ & $\begin{array}{l}S_{2, \mathrm{~m}}^{\mathrm{f}} \\
(M / 1)\end{array}$ \\
\hline Hydrocortisone & 293 & 3633 & 12.4 & 8.1 & $5.8 \times 10^{8}$ & $9.4 \times 10^{7}$ \\
21-acetate & 313 & 3381 & 12.4 & 7.1 & $4.0 \times 10^{-8}$ & $6.5 \times 10^{-7}$ \\
21-propionate & 329 & 4014 & 12.2 & 5.1 & $2.9 \times 10^{-7}$ & $4.7 \times 10^{-6}$ \\
21-butyrate & 345 & 4147 & 12.0 & 5.1 & $6.3 \times 10^{-7}$ & $1.0 \times 10^{-5}$ \\
21-valerate & 362 & 4280 & 11.8 & 4.6 & $1.4 \times 10^{-6}$ & $2.2 \times 10^{-5}$ \\
21-hexanoate & 378 & 4413 & 11.7 & 4.1 & $4.4 \times 10^{-6}$ & $7.0 \times 10^{-5}$ \\
21-heptanoate & 394 & 4546 & 11.5 & 2.5 & $1.6 \times 10^{-5}$ & $2.5 \times 10^{-4}$ \\
\hline
\end{tabular}

${ }^{8}$ Estimated molar volume. For hydrocortisones see Ref. [6]. Others based on $293 \mathrm{~cm}^{3}$ for hydrocortisone and partial molar volumes of added/subtracted functional groups [22].

${ }^{b}$ Molar attraction constant. For hydrocortisone $\left(E V_{\mathrm{m}}\right)^{1 / 2}-\delta_{2} V_{\mathrm{m}}$. For the esters estimated from hydrocortisone's molar attraction constant and function group molar attraction constants of Hoy [21]. For example, for the acetate ester:

$\left(E V_{\mathrm{m}}\right)^{1 / 2}=\left(E V_{\mathrm{m}}\right)_{\text {hydrocortisone }}^{1 / 2}-\left(E V_{\mathrm{m}}\right)_{\text {hydroxyl }}^{1 / 2}+\left(E V_{\mathrm{m}}\right)_{\text {ester carboxyl }}^{1 / 2}+\left(E V_{\mathrm{m}}\right)_{\text {methyl group }}^{1 / 2}$ or $(E V)_{\text {h.c. acetate }}^{1 / 2}=3633-226+327+148$.

'Solubility parameter of solute. For hydrocortisone see Ref. [6]. For the esters calculated from $\delta_{2}=\sum\left(E V_{\mathrm{m}}\right)^{1 / 2} / V_{\mathrm{m}}$. The $\sum\left(E V_{\mathrm{m}}\right)^{1 / 2}$ is done as in footnote (b).

${ }^{\mathrm{d}}$ Estimated from known mole fraction solubilities in hexane at $25^{\circ} \mathrm{C}$, estimated solubility parameters, experimentally determined melting points and estimated molar volumes using a solubility parameter for hexane of $7.3\left(\mathrm{cal} / \mathrm{cm}^{3}\right)^{1 / 2}$. Sulubilities are low and the volume fraction of the solvent in the calculation is set at unity with little error.

${ }^{\mathrm{e}}$ Mole fraction solubility in polydimethylsiloxane calculated from the regular solution equation $\ln x_{2}=\Delta H_{\mathrm{f}}\left(T_{\mathrm{f}}-T\right) / R T_{\mathrm{f}} T-\left(\delta_{1}-\delta_{2}\right)^{2} V_{\mathrm{m}} / R T$.

${ }_{\mathrm{f}}$ Molar solubility in polydimethylsiloxane calculated from eqn. (11) (see text).

Overall, the alkyl chain length influence upon permeability of silicone rubber within the hydrocortisone series has been shown to be in striking parallel with hexane/water solubility ratios. By analogy, it is apparent that the partitioning step between water and membrane is principally responsible for the permeability coefficient pattern. Apparently diffusivities of the homologs within the membrane do not vary greatly, which is as expected. Despite the size of the molecules, the hydrodynamic boundary layer resistance becomes a rate controlling factor for the higher chain length esters, the effect being especially pronounced for the heptanoate. A potentially important observation relevant to topical administration of these compounds is the large increase in steadystate flux from saturated solutions. The destabilization of the crystalline structure with increasing length of the alkyl chain leads to increases in solubility in the nonpolar polymer matrix and, correspondingly, increases in flux. Melting points of hydrocortisone 21-esters increase beyond a chain length of seven and on this basis alone one can confidently predict that the solubilities of the hex- 


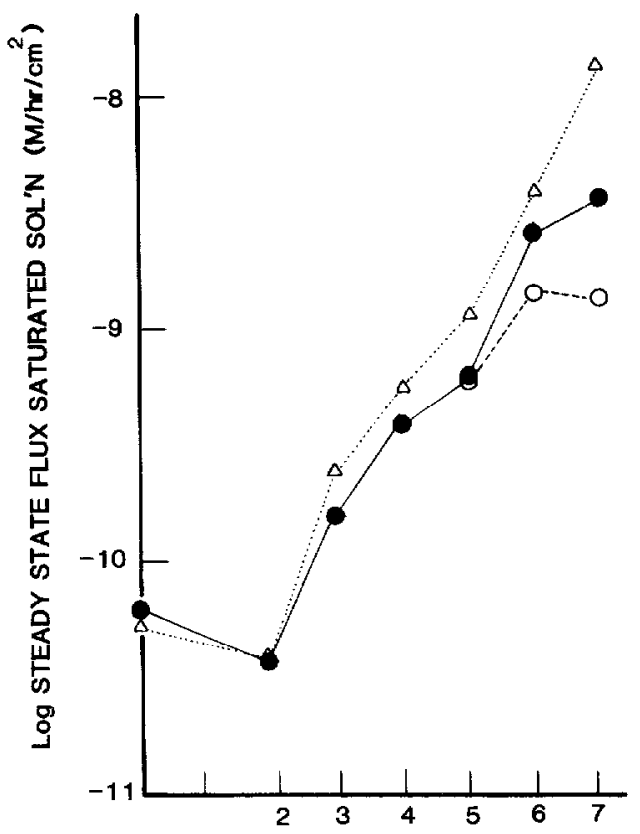

ALKYL CHAIN LENGTH

Fig. 7. Semilogarithmic plot of boundary layer corrected flux from saturated solutions versus alkyl chain length of the hydrocortisone 21 -esters. Hydrocortisone is arbitrarily plotted at $n=0$. The open circles are the actual experimental values. Measurable boundary layer influence is evident for the valerate through heptanoate esters. The open triangles are permeability coefficients calculated from regular solution theory estimates of solubility in the silicone rubber. These are normalized to the acetate. It can be seen that the entire profile can be well approximated theoretically as long as there is one defined point (acetate in this instance). The gap between predicted and actual values widens as the alkyl chain length is increased. This is believed to be mostly the result of additive error in estimating the solubility parameters of the esters.

anoate and heptanoate esters in the silicone rubber matrix are maximum within the homologous series. This point, coupled with the transition to boundary layer control as the alkyl chain length approaches seven, means that the hexanoate and heptanoate esters of hydrocortisone have the highest transport rates from saturated solutions within the series. It is expected but remains to be shown that advantageously high fluxes of these compounds can be achieved through skin.

\section{References}

1 G.L. Flynn, A structural approach to partitioning - Estimation of steroid partition coefficients based upon molecular constitution. J. Pharm. Sci., 60 (1971) 345-353. 
2 G.L. Flynn and S.H. Yalkowski, Correlation and prediction of mass transport across membranes. I: Influence of alkyl chain length on the flux determining properties of barrier and diffusant, J. Pharm. Sci., 61 (1972) 838-851.

3 S.H. Yalkowski and G.L. Flynn, Transport of alkyl homologs across synthetic and biological barriers: A new model for chain length-activity relationships, J. Pharm. Sci., 62 (1973) 210-217.

4 S.H. Yalkowski and G.L. Flynn, Correlation and prediction of mass transport across membranes. II: Influence of vehicle polarity on flux from solutions and suspensions, J. Pharm. Sci., 63 (1974) 1276-1280.

5 H.H. Duriheim, G.L. Flynn, W.I. Higuchi and C.R. Behl, Permeation of hairless mouse skin. I: Experimental methods and comparison with human epidermal permeation by alkanols, J. Pharm. Sci., 69 (1980) 781-786.

6 T.A. Hagen and G.L. Flynn, Solubility of hydrocortisone in organic and aqueous media: Evidence for regular solution behavior in apolar solvents, J. Pharm. Sci., 72 (1983) 409-414.

7 C.R. Behl, G.L. Flynn, T. Kurihara, N. Harper, W.M. Smith, W.I. Higuchi, N.F.H. Ho and C.L. Pierson, Hydration and percutaneous absorption. I: Influence of hydration on alkanol permeation through hairless mouse skin, J. Invest. Dermatol., 75 (1980) 346-352.

8 C.R. Behl, E.E. Linn, G.L. Flynn, C.L. Pierson, W.I. Higuchi and N.F.H. Ho, Permeation of skin and eschar by antiseptics. I: Baseline studies with phenol, J. Pharm. Sci., 72 (1983) 391-397.

9 T. Higuchi, Design of chemical structure for optimal dermal activity, Curr. Prob. Dermatol., 7 (1978) 121-131.

10 G.L. Flynn, N.F.H. Ho, S. Hwang, E. Owada, A.M. Molokhia, C.R. Behl, W.I. Higuchi, T. Yotsuyanagi, Y. Shah and J.Y. Park, Interfacing matrix release and membrane absorption - Analysis of steroid absorption from a vaginal device in rabbit doe, in: D.R. Paul and F.W. Harris (Eds.), Controlled Release Polymeric Formulations, ACS Symposium Series No. 33, 1976, Chap. 7, pp. 87-122.

11 T.J. Roseman, Release of steroids from a silicone polymer, J. Pharm. Sci, 61 (1972) 46-50.

12 G.L. Flynn and T.J. Roseman, Membrane diffusion. II: Influence of physical absorption on molecular flux through heterogeneous dimethylpolysiloxane barriers, J. Pharm. Sci., 60 (1971) 1788-1796.

13 D.R. Paul and D.R. Kemp, The diffusion time lag in polymer membranes containing adsorptive fillers, J. Polym. Sci., Symp., (41) (1973) 79-93.

14 W.I. Higuchi and T. Higuchi, Theoretical analysis of diffusional movement through heterogeneous barriers. J. Amer. Pharm. Assoc., Sci. Ed., 49 (1960) 598-606.

15 C.F. Most, Jr., Some filler effects on diffusion in silicone rubber, J. Appl. Polym. Sci., 14 (1970) 1019-1024.

16 G.L. Flynn, S.H. Yalkowski and T.J. Ruseman, Mass transport phenomena and models: Theoretical aspects, J. Pharm. Sci, 63 (1974) 479-510.

17 S.H. Yalkowski, G.L. Flynn and T.G. Slunick, The influence of chain length on the physicochemical and crystalline properties of organic homologs, J. Pharm. Sci., 61 (1972) 852-857.

18 G.L. Flynn, A.B. French, N.F.H. Ho, W.I. Higuchi, E.H. Ostafin, L.H. Warbasse, G.F. Amidon and E. Williams, Some hydrodynamic boundary layer influences on mass transfer coefficients, J. Membrane Sci., 19 (1984) 289-308.

19 A.S. Michaels, P.S.L. Wong, R. Prather and R.M. Gale, A thermodynamic model of predicting the transport of steroids in polymer matrices, AIChE J., 21 (1975) 1073-1080.

20 P.A. Small, Some factors affecting the solubility of polymers, J. Appl. Chem., 3 (1975) 71-80.

21 L.L. Engle, Physical Properties of Steroid Hormones, Macmillan, New York, NY, 1963.

22 K.L. Hoy, New values of the solubility parameters from vapor pressure data, J. Paint Technol., 42 (1970) 1-8. 